Article

\title{
Structural Elucidation of $\alpha$-Cyclodextrin-Succinic Acid Pseudo Dodecahydrate: Expanding the Packing Types of $\alpha$-Cyclodextrin Inclusion Complexes
}

\author{
Sofiane Saouane and Francesca P. A. Fabbiani * \\ Received: 16 November 2015; Accepted: 16 December 2015; Published: 24 December 2015 \\ Academic Editor: Sławomir J. Grabowski \\ Georg-August-Universität Göttingen, GZG, Abt. Kristallographie, Goldschmidtstr. 1, 37077 Göttingen, \\ Germany; ssaouan@gwdg.de \\ * Correspondence: ffabbia@gwdg.de; Tel.: +49-551-39-33935; Fax: +49-551-39-9521
}

\begin{abstract}
This paper reports a new packing type of $\alpha$-cyclodextrin inclusion complexes, obtained here with succinic acid under low-temperature crystallization conditions. The structure of the 1:1 complex is characterized by heavy disorder of the guest, the solvent, and part of the host. The crystal packing belongs to the known channel-type structure; the basic structural unit is composed of cyclodextrin trimers, as opposed to the known isolated molecular or dimeric constructs, packed along the $c$-axis. Each trimer is made of crystallographically independent molecules assembled in a stacked vase-like cluster. A multi-temperature single-crystal X-ray diffraction analysis reveals the presence of dynamic disorder.
\end{abstract}

Keywords: cyclodextrins; inclusion complexes; crystallization; structural disorder

\section{Introduction}

Native cyclodextrins (CDs) are cyclic oligosaccharides with low chemical toxicity [1,2]. They are formed by several $\alpha$-D-glucopyranose units, linked by $\alpha(1 \rightarrow 4) O$-glycosidic bonds. A CD molecule has a hollow truncated cone-like shape where the primary hydroxy groups sit on the narrow side and the secondary ones on the wide side of the truncated cone. The architecture of CD molecules favors the partition of the structure into an outer surface and an inner cavity, which are hydrophilic and hydrophobic in character, respectively [3]. This molecular feature boosted the interest of using CDs as "molecular cages" [4] in both the solid and solution states, particularly in the pharmaceutical field where CDs are known to improve the aqueous solubility, and thus bioavailability, of poorly soluble active pharmaceutical ingredients [3]. The formation of CD inclusion complexes in the solid state, particularly with organic molecules, has been pioneered by Saenger [5-7] and Harata [8]. Three packing types have been described for both $\mathrm{CD}$ hydrates and inclusion complexes: two of these belong to the cage type and are known as herringbone- and brick-type packing, and the third one is the channel type [7].The packing preference of $\mathrm{CD}$ molecules for one packing type is closely related to the size and shape of the guest molecule [7].

The work presented here is part of ongoing investigations of cyclodextrin inclusion complex and hydrate formation at both ambient- and high-pressure crystallization conditions using water as crystallization medium [9]. $\alpha$-CD (Figure 1), the smallest natural cyclic oligosaccharide with six sugar units, was chosen for studying inclusion complex formation with small molecules (here defined as molecules with a molecular weight $<500$ Daltons), particularly to compare and contrast the effects of non-ambient (low-temperature and high-pressure) crystallization conditions on complex formation. A Cambridge Structural Database [10] (CSD) search (the CSD, V 5.36 including updates to November 2014 was searched for structures with 3-D coordinates) indicates that $\alpha$-CD crystallizes 
in the channel-packing type in $52 \%$ of the total 96 hits, followed by the brick-type in $26 \%$ and the herringbone-type in $22 \%$ of the structures. In the channel-packing type favored by $\alpha-C D$, guest molecules are inserted lengthwise into the host's cavity, forming infinite columns.

(a)

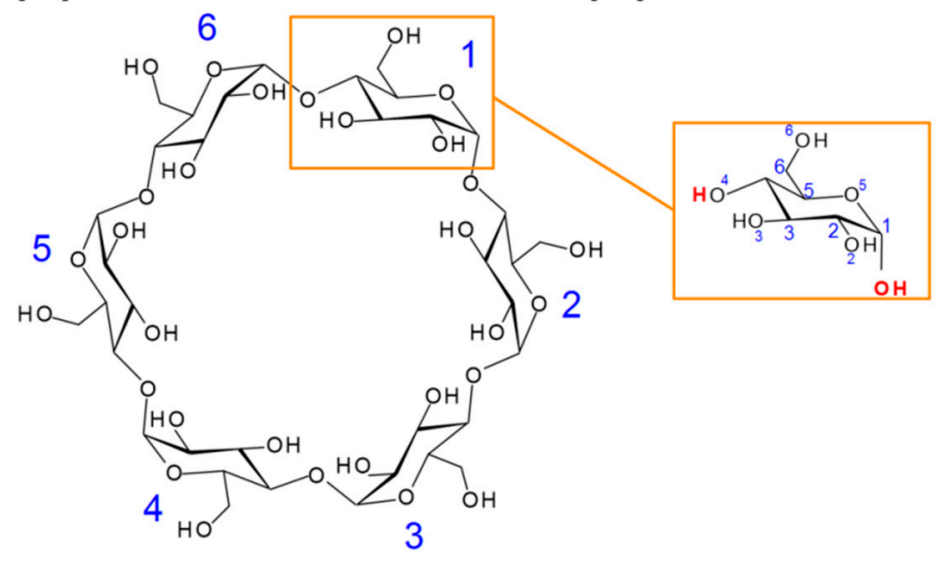

(c)

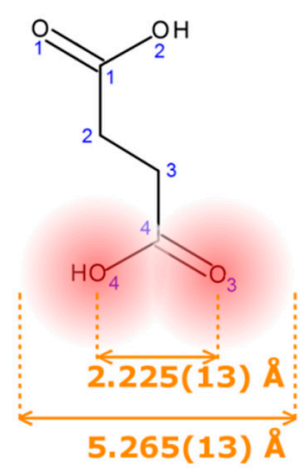

Figure 1. Diagrams and numbering schemes of: (a) $\alpha$-cyclodextrins; (b) $\alpha$-D-glucopyranose; (c) succinic acid (SA): the mean distance $\mathrm{O}(3)-\mathrm{O}(4)$ was computed from 72 structures of SA in the Cambridge Structural Database (CSD), the width of SA is the (mean distance $\mathrm{O}(3)-\mathrm{O}(4)$ plus twice the van der Waals radius of an oxygen atom.

Succinic acid (SA, Figure 1), an aliphatic dicarboxylic acid, is essential in aerobic cellular metabolism by intervening in the citric acid cycle, a metabolic pathway for the regeneration of adenosine triphosphate (ATP), which is the main energy source of most cellular functions [11]. SA is a FDA-Generally Recognized As SAFE (GRAS) substance also used in the pharmaceutical industry for the preparation of succinate ester derivatives of active pharmaceutical ingredients. The structure of a $\beta$-CD.SA inclusion complex (CSD refcode KIJSEC) has been previously obtained while investigating the enhancement of succinic anhydride's reactivity using $\beta-C D$ as molecular cages in aqueous solutions [12]. We found that this complex can easily be obtained with SA instead of succinic anhydride; the large cavity size of $\beta-C D$, which is $6.0-6.5 \AA$ in diameter [13], can easily accommodate SA and the crystal structure of the complex shows several intermolecular interactions between host, guest and solvent molecules [12]. We hypothesize that with a width (5.265(13) $\AA$, see Figure 1) commensurable with the cavity diameter of $\alpha$-CD (4.7-5.3 $\AA$ ) [13], SA could in principle form a crystalline complex with $\alpha$-CD. The literature shows that similar linear compounds form inclusion complexes with $\alpha-C D$ (see for example CSD refcodes BUPDEV [14], CDKABA [15] and XIGBOE [16]).

\section{Results and Discussion}

Low-temperature crystallization of an equimolar mixture of $\alpha$-CD with SA in water led to the formation of hexagonal prism shaped crystals. A first polarized microscopy analysis of the crystals through the hexagonal face indicated an absence of light extinction characteristic of uniaxial crystals. This microscopic analysis endorsed the choice of the unit cell from X-ray diffraction, with successful indexing of the reflections using a rhombohedral unit cell. The reflections could also be indexed using a lower symmetry monoclinic unit cell, through the transformation matrix $(-1 / 3-2 / 3-2 / 3,100,-1 / 3$ $-2 / 31 / 3$ ). The choice of a trigonal crystal system dictates that both host and guest molecules sit on a 3 -fold rotation axis going through $\alpha$-CD cavities. Heavy disorder of the guest, evident from the electron density maps, could be modelled in the higher symmetry space group and this was finally chosen for refinement. 
The $\alpha$-CD.SA 1:1 inclusion complex crystallizes in space group R32 with $3 \times 1 / 3 \alpha$-CD molecules in the asymmetric unit, here named A, B and C (Figure 2). Each $\alpha$-CD sits on the 3-fold rotation axis and encloses one SA molecule disordered over six positions; crystallographically, this is modelled by having two SA molecules per cavity each with $1 / 6$ site occupancy. $\alpha$-CD C is further disordered over two positions with $2 / 3(C)$ and $1 / 3\left(C^{\prime}\right)$ site occupancies. In addition the unit cell contains $c a$. 12 water molecules making the complex a pseudo dodecahydrate.

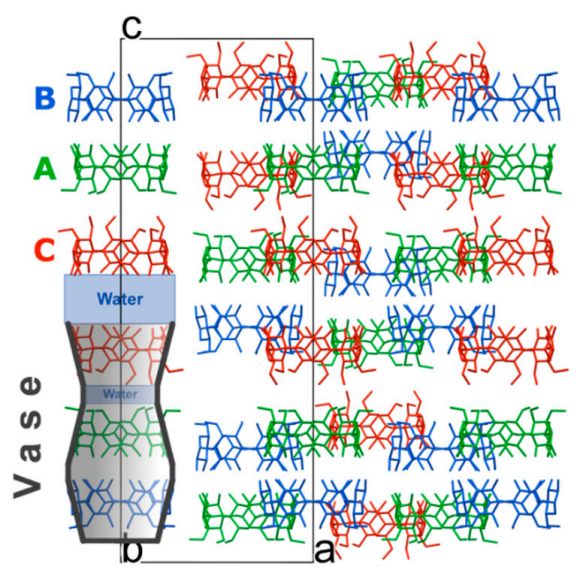

Figure 2. Crystal packing of $\alpha$-CD molecules viewed along the $b$-axis. $H$ atoms, disorder of $\alpha-C D$ $\mathrm{C}$, SA and water molecules have been omitted for clarity. Symmetry-equivalent molecules are color coded.

In the solid state $\alpha$-CD molecules have always been known, to the best of our knowledge, to pack as either distinct entities or dimers [6,8]. In this work, a new building block is observed, namely $\alpha-C D$ trimers packed along the $c$-axis. Each trimer is made of crystallographically independent molecules assembled in a stacked vase-like cluster (Figure 2). The stacked trimer motif is not unknown for $\gamma-C D$ molecules (see for example CSD refcodes FEJFIJ, FEJFOP, NUNRIX, SIBJAO, SIBJES) [17-19]; however the concept of vase-like packing has not been previously reported. It has also been reported that $\beta$-CD molecules crystallize as trimers (CSD refcodes RIPKIL, OCIGAK) [20,21] or tetramers [22,23]. The structure of the title compound appears to be very similar to that of $\alpha$-CD.hexa-ethylene glycol reported by Harada et al. (CSD refcode LOJTUZ, no 3D coordinates deposited in the CSD) [24]; however, this publication neither describe the distinctive trimer arrangement in detail, nor identifies a new packing type. In contrast, in a conference abstract, Caira et al. have recently reported that the $\alpha$-CD-lipoic acid system "crystallizes in the trigonal system, space group R32, with three independent CD molecules in the asymmetric unit and is not isostructural with any known CD complex" [25]. These observations make the reported packing type rare but not unknown.

The vase-like cluster in the $\alpha$-CD.SA inclusion complex is formed by two sub-dimers: (1) a head-to-head dimer, which is stabilized by $\mathrm{H}$-bonds between secondary hydroxy groups of $\alpha$-CDs $\mathrm{A}$ and $\mathrm{B}$; and (2) a tail-to-tail dimer between $\alpha$-CDs $\mathrm{A}$ and $\mathrm{C}$ interconnected through a cluster of water molecules. Analysis of planes formed by glycosidic O-atoms shows that the interplanar distance in the head-to-head dimer is $7.022 \AA$, compared to a mean distance of $9.060 \AA$ for the tail-to-tail dimer (Figure 3). Successive vase-like clusters, stacked via two-fold rotation symmetry, are separated by a layer of water molecules forming a complex H-bonded network that holds two vase-like structures together.

The three $\alpha$-CD molecules forming the vase-like cluster exhibit an almost ideal cylindrical shape and form infinite linear channels extending along the $c$-axis of the unit cell in a honeycomb-type arrangement. A honeycomb or quasi-hexagonal pattern ensures effective close packing [19,26], and for CDs it was reported for the first time by Saenger in 1980, while describing the general arrangement of CDs in crystals, as "hexagonal packing of stacks" [5]. A careful analysis of the reported channel-type 
structures of $\alpha$-CD inclusion complexes, including hydrates, in the CSD shows that honeycomb packing is observed in 27 out of 50 structures (CSD refcodes of these structures are reported in the Supplementary Information), though this particular structural feature has been rarely reported explicitly. Instead, authors usually report the type of $\alpha$-CD dimer arrangement, head-to-head or head-to-tail, in channel-type structures.

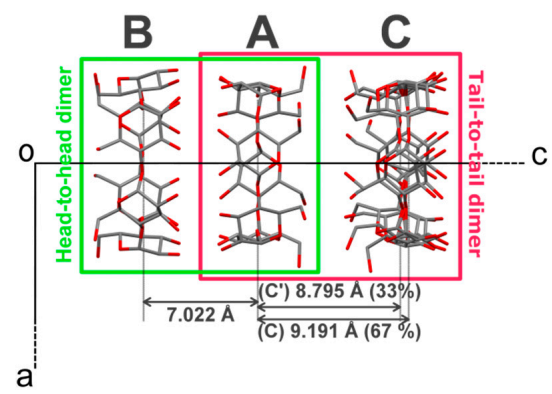

Figure 3. Projection of $\alpha$-CD.SA structure along the $b$-axis. The crystallographically-independent CD molecules are named A, B and C, see main text for details. H atoms, SA and water molecules have been omitted for clarity. The distances refer to gap in $\AA$ between the least-square planes formed by all $\mathrm{O}(4)$ atoms involved in the glycosidic bonds.

One of the features of $\mathrm{CDs}$ is the flexibility of the primary-hydroxy groups. The conformation of the hydroxy group is defined by the value of the $\mathrm{O}(5)-\mathrm{C}(5)-\mathrm{C}(6)-\mathrm{O}(6)$ torsion angle (Figure 1): a preferred (-)-gauche conformation in which the hydroxy group is facing the exterior of the cavity, and a less preferred (+)-gauche conformation where the hydroxy group is facing the inner cavity [27]. All but one primary hydroxy groups in $\alpha-\mathrm{CD}$ exhibit the (-)-gauche conformation and are directed away from the cavity. The rotation of the primary hydroxy group to the less favored (+)-gauche conformation in molecule $\mathrm{C}$ can be explained by the formation of the short H-bonds [2.52(4) and 2.79(1) $\AA$ ] with a disordered water molecule (named O(10)_91 in the structure). A CSD search (V 5.36) shows that (-)-gauche conformers are not unknown for $\alpha$-CD inclusion complexes and indicate the absence of direct $\mathrm{H}$-bonds between the guest and the host molecules, which could explain the high degree of rotational disorder of the guest observed here. In contrast, in the structure of $\beta$-CD.SA which contains one full guest and host molecules in the asymmetric unit, SA is H-bonded to two primary $C D$ hydroxy groups, which are facing the inner cavity, through one water molecule each. Interestingly, the SA molecule in the $\beta$-CD.SA inclusion complex does not exhibit disorder.

A search in the CSD (V 5.36 including updates to November 2014) based on the $C(1)-C(2)-C(3)-C(4)$ torsion angle of the guest (Figure 1) shows that SA molecules exhibit two conformations in the solid state, trans and gauche, with an incidence of 90 and $10 \%$, respectively, out of a total of 165 structures. Lisnyak et al. reported that the trans conformer is energetically more favorable than the gauche one with an energy difference of $31.4 \mathrm{~kJ} \cdot \mathrm{mol}^{-1}$ [12]. In $\beta$-CD.SA (CSD refcode KIJSEC [12]), SA molecules lie almost equatorially in the $\beta$-CD cavity and exhibit the gauche conformer. In contrast, the electron density maps of $\alpha$-CD.SA point to the trans conformation with SA molecules extended along the $c$-axis. The difference in conformation may be directly related to the cavity size differences between $\alpha$ - and $\beta-C D$, with an axial inclusion mode being more commensurable with the size of the $\alpha$-CD cavity. Although as a result of this one might expect a tighter fit of $S A$ inside $\alpha-C D$, the guest is actually found be disordered. This is in line with the almost ideal cylindrical shape of the host, yielding a more evenly distributed cavity space, and the absence of direct H-bonds between the guest and host molecules. In $\alpha$-CD.SA the axial inclusion mode is described by the almost orthogonal angular differences between the planes formed by the glycosidic O-atoms of the ordered CD molecules $\mathrm{A}$ and $\mathrm{B}$ and to the planes formed by the SA molecules inside the respective cavities (SA planes were calculated using $C(1)-C(2)-C(3)-C(4)$ ). For $\alpha-C D C$, similar calculations show that the same planes are 13 to $18^{\circ}$ off from being orthogonal. The different 
inclusion modes of SA inside $\alpha$ - and $\beta-\mathrm{CD}$ are also associated with different crystal packing: the trans SA molecules in $\alpha$-CD.SA form a H-bonded linear chain (Figure 4) that is associated with the formation of a channel-type packing. On the other hand, in the $\beta$-CD.SA complex gauche SA molecules are "buried" in the cavities and the herringbone-type packing observed in the hydrated $\beta-C D$ and small-guest molecules structures is conserved. This comparative analysis based on size, shape and functionality of the guest molecule supports the work by Saenger and Steiner [7].

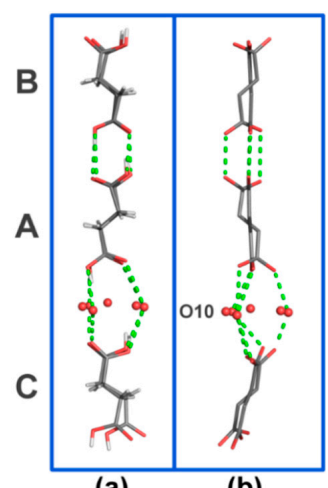

(a) (b)

Figure 4. H-bonded motif formed by SA molecules in the asymmetric unit viewed (a) along the $a$-axis and (b) along the $b$-axis. $\mathrm{O}-\mathrm{O}$ contacts are represented by dashed green lines. $\mathrm{H}$-atoms (in $\mathrm{b}$ ), $\alpha$-CD and water molecules have been omitted for clarity. SA molecules are contained in the cavities of the crystallographically-independent CD molecules named A, B and C, see main text for details.

\subsection{Insight into Disorder}

In an attempt to gain a better insight into the disorder of $\alpha$-CD.SA inclusion complex and to investigate whether a temperature-dependent phase transition takes place, we extended our initial work to a multi-temperature investigation covering the 100-270 K temperature range, in an ascending temperature ramp.

The crystallographic data of all structures, summarized in Table S1, show small differences in the lattice constants across the temperature range. The lattice parameters were normalized to the unit cell values of the $100 \mathrm{~K}$ crystal structure and are visually represented in Figure 5. Approximately uniform thermal expansion up to $180 \mathrm{~K}$ is observed for both the $c$ - and $a$-axes. For the unit cell volume, the rate of thermal expansion is approximately linear up to this temperature. From 180 to $240 \mathrm{~K}$, the complex overall expands but the $c$-axis shows negative thermal expansion. The negative thermal expansion for this axis is marked between 240 and $270 \mathrm{~K}$ so that a slight volume contraction is concomitantly observed.

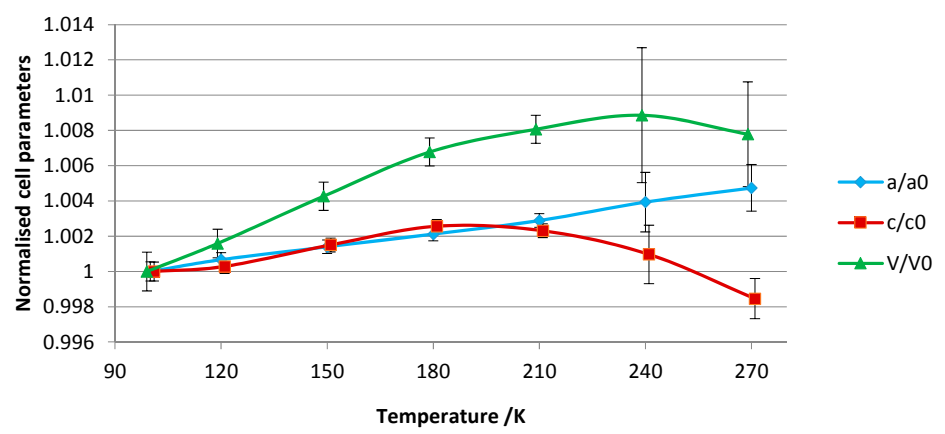

Figure 5. The evolution of the normalized unit-cell parameters as function of temperature (errors at $3 \sigma$ level) taken from the structures in Supplementary Table S1, see Supplementary Information for details. 
The variations in the unit-cell parameters can be related to the following structural features: (1) lengthening of the $a$-axis $(a=b)$ as a function of increasing temperature is associated with lengthening of the intermolecular contacts between adjacent $\alpha-C D$ molecules along the same axis; (2) the negative thermal expansion along the $c$-axis is associated with a compression of the vase-like cluster (see Figure S1), mainly arising from compression of the thin water layer that stabilizes the $\alpha-\mathrm{CD}$ A-C tail-to-tail dimer (Figure 2). This thin water layer is formed by one disordered water molecule (named $\mathrm{O}(10) \_91$ in the structure), and its symmetry equivalents, H-bonded to the (+)-gauche primary hydroxy group of $\alpha$-CD molecule C (Figure 4).

An apparent ordering of $\alpha-C D C$ is observed at $270 \mathrm{~K}$ although it should be noted that the structure has large temperature factors. In fact, the low-temperature study points to the presence of dynamic disorder, whereby low temperatures freeze the movement of $\alpha-C D C$ into two distinct positions. Ordering at $270 \mathrm{~K}$ is associated with a rearrangement of hydroxy groups: $\alpha-C D C$ has two (+)-gauche primary hydroxy conformers, instead of one observed at low temperature. The new (+)-gauche conformer, pointing towards the inner cavity, is $\mathrm{H}$-bonded to the disordered water molecule $\mathrm{O}(10) \_91$ with a distance of 2.52(9) $\AA$. This indicates that there is interplay between movement of $\alpha-\mathrm{CD}$ and water molecules towards stabilization of the structure. The multi-temperature experiment did not provide information on the nature of the disorder of the guest molecules, for which it is likely that a combination of dynamic and static disorder is present.

The $100 \mathrm{~K}$ structural model was refined based on the atomic coordinates of the $90 \mathrm{~K}$ model, and was then used as a starting model for all other temperature points. Modeling of disordered water molecules in a highly hydrated structure is subject to user bias. Temperature factors and site occupancy parameters suffer from correlation and in the absence of independent information, e.g., on water content determined by thermal analysis, the user must make judicious use of refinement tools in order to obtain a reasonable model, which does not necessarily lead to the lowest $R$-factor. Comparing Fourier and difference Fourier maps can give an indication on the mean position and site occupancy of the atoms provided a consistent data reduction and refinement strategy for all data points is applied. To minimize model bias and double check the stability of the model, the SHELXL WIGL command was used as a cross-validation method during the refinement [28]. Two sets of results were then generated. In the first set, all water molecules were placed and refined according to the difference Fourier electron density maps (results reported in Table S1). In the second set, all water molecules were omitted during refinement in order to compare the evolution of the maps as a function of increasing temperature. The latter set of structures can be used to emphasize the change in the positions of water molecules and site occupancies by two methods:

(a) An analysis with SHELXLE [29] allows a qualitative analysis of the changes in the difference density as a function of temperature. Figure 6 clearly illustrates the loss of this signal from the solvent region as temperature is increased.

(b) Void analysis performed with MERCURY [30] using a probe radius of $1.2 \AA$ and a grid spacing of $0.2 \AA$, calculated using the contact surface algorithm, reveals that the volume occupied by the solvent does not change significantly as temperature is increased (Figure S2).

An analysis, based on the fully refined structures, of the anisotropic displacement parameters (ADP) as function of temperature indicates larger thermal motion at higher temperatures, as expected (see Figure S3). Analysis of the refined $U_{\mathrm{ij}}$ values of $\mathrm{O}(1) \rightarrow \mathrm{O}(6)$ water molecules, which are located in the space between adjacent $\alpha-C D$ columns (Figure 2), shows comparable expansion of the three diagonal elements $U_{11}, U_{22}, U_{33}$ of the ADP tensor. These elements are parallel to the reciprocal unit-cell axes $a^{*}, b^{*}$ and $c^{*}$, respectively, and in the case of a rhombohedral crystal system, $U_{33}$ is parallel to the $c$-axis. For $\alpha$-CD atoms, the thermal expansion of $U_{33}$ is much more pronounced compared to the two other directions. Hence, while water molecules increase their vibrations in all directions as temperature increases, $\alpha$-CD molecules have more freedom along the $c$-direction. 


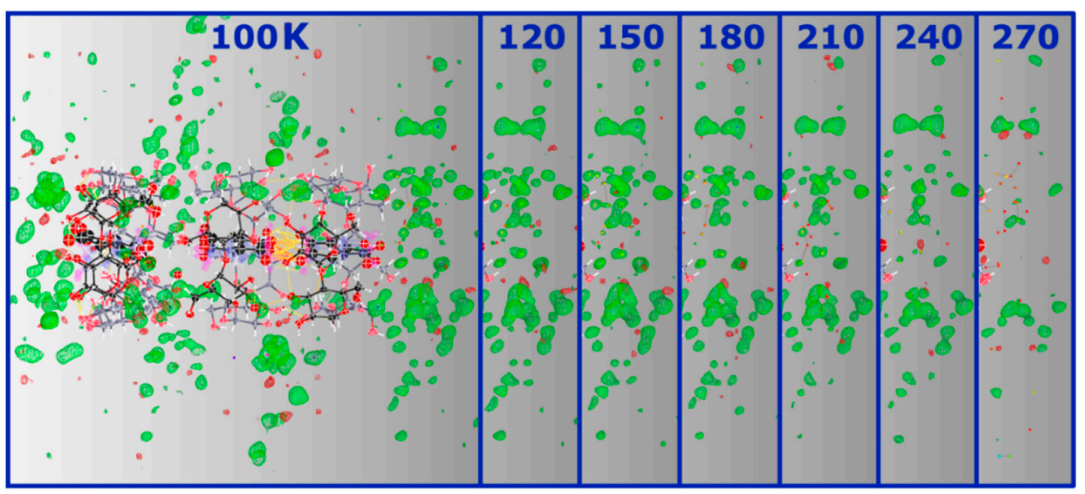

Figure 6. SHELXLE $F_{\text {obs }}-F_{\text {calc }}$ maps (at $0.40 \mathrm{e}^{-} / \AA^{3}$ ) of $\alpha$-CD.SA inclusion complex at $100 \mathrm{~K}$ and the respective solvent region at the bottom (right-hand side in the picture) of the vase-like cluster at the different temperatures showing the decrease of the signal as a function of increasing temperature. A similar decrease, not shown here, is observed for the other side.

To summarize, the loss of diffraction quality at higher temperatures can be directly ascribed to structural disorder, primarily of the solvent region. It is not unlikely that at even higher temperatures, water molecules vibrate excessively leading to disruption of the crystal packing and structural instability as testified by complete loss of diffraction at $298 \mathrm{~K}$. Given the channel-type packing adopted by the structure, it is conceivable that water molecules find their way out of the structure. Moreover, the multi-temperature study also shows that the guest molecules inside $\alpha$-CD cavities are freely rotating with no apparent ordering induced by lowering the temperature. This is due to the lack of $\mathrm{H}$-bond interactions between the guest and the host molecules, which, in theory, should help to stabilize the position of the guest molecules. A CSD search reveals that the lack of direct intermolecular contacts between host and guest molecules is not unusual for CD inclusion complexes, and that the guest is indirectly H-bonded to the host via water molecules.

\subsection{High-Pressure Crystallization Results}

We have investigated complex formation at high pressure up to $0.7 \mathrm{GPa}$ and in the 277-293 K temperature range. Two avenues were explored: first, a diamond-anvil cell (DAC) was loaded with a saturated 1:1 aqueous solution of $\alpha-\mathrm{CD}$ and SA and pressure was increased to induce precipitation. No crystals were obtained in this manner. In a second set of experiments, a single crystal of the $\alpha$-CD.SA complex crystallized at ambient-pressure conditions was loaded in a DAC together with either its mother liquor or water. Pressure was increased and gradual dissolution of the crystal was observed, similar to what was reported for pure $\alpha$-CD hydrate form I [9]. Subsequent to dissolution, no crystallization event was recorded. Upon releasing pressure, there was no microscopic evidence for crystallization of either SA or $\alpha-C D$ alone. While our experiments were not comprehensive, the lack of complex formation at high pressure may be ascribed to concentration, the formation of a particularly stable complex in solution stabilized at high pressure and kinetic effects; it is also conceivable that structural disorder is essential for stability of the $\alpha$-CD.SA complex (the entropic contributions of structural disorder in molecular compounds have for instance been discussed in [31-34]) and that high pressure, favoring more dense, and in general more ordered states, hinders the in situ formation of a complex.

\section{Experimental Section}

\subsection{Ambient-Pressure Crystallization}

Both $\alpha-C D$ and SA were bought from Fluka (Munich, Germany ) and SIGMA (Munich, Germany), respectively, and used without further treatment. An undersaturated solution with a 1:1 
molar ratio mixture of $\alpha$-CD (194.57 mg) and succinic acid $(23.6 \mathrm{mg})$ was prepared in approximately $2 \mathrm{~mL}$ of demineralized water. Fast evaporation of the solution yielded a glass at ambient conditions. A combination of cold temperatures ( $c a .277 \mathrm{~K}$ ) and very slow evaporation over the course of six months led to the crystallization of hexagonal prism-shaped crystals. When the experiment was repeated using a more concentrated solution, crystallization occurred within a week at the same low-temperature conditions.

\subsection{High-Pressure Crystallization}

The high-pressure crystallization experiment was carried out using an in-house modified Merrill-Bassett DAC [35] with a half opening angle of $45^{\circ}$. The DAC was equipped with $800 \mu \mathrm{m}$ culet diamonds of low fluorescence grade and Inconel gaskets with a starting diameter hole of $350 \mu \mathrm{m}$. Pressure was monitored using the ruby fluorescence method described by Piermarini et al. [36].

\subsection{Data Collection and Reduction}

Several crystals were tested on the diffractometer. When left out of the mother liquor, crystals were found to be unstable at room-temperature conditions, yielding poor diffraction quality even when measured at low temperature. Maintaining low temperature from the moment the crystal was extracted from the mother liquor until final mounting on a glass fiber on the goniometer's head was essential to ensure good diffraction quality: in order to achieve this a drop containing mother liquor and crystals was pipetted onto a microscope slide that had previously been cooled using an ice bath beneath the slide. Subsequently, the crystal was moved from the mother liquor to a cold drop of mounting oil and then to the goniometer's head under a cold and dry-nitrogen stream [37].

Data collection was undertaken using a Bruker-AXS APEX II diffractometer (Bruker-AXS, Karlsruhe, Germany) equipped with graphite-monochromatic Mo-K $\alpha$ radiation and an Oxford Cryosystems low-temperature device (Oxoford Cryosystems Ltd., Oxford, UK). X-ray data were first collected on a single crystal specimen at $90 \mathrm{~K}$. A subsequent experiment was performed at low-temperature conditions on a second single-crystal specimen. Data sets were collected on the same single crystal using the same data collection strategy at 100, 120, 150, 180, 210, 240 and $270 \mathrm{~K}$. The purpose of this experiment was to monitor structural changes, e.g., phase transitions, and possible crystal decay as function of temperature.

Data integration and global-cell refinement were performed with the program SAINT (Bruker-AXS, Madison, WI, USA) [38]. Absorption correction was performed with SADABS (Bruker-AXS, Madison, WI, USA) [39]. Structure solution of this medium-sized structure was based on Patterson-seeded dual-space recycling in the SHELXD program (Göttingen, Germany) [40]. Structures were refined by full-matrix least squares against $F^{2}$ using SHELXL-2014/7 (Göttingen, Germany) [41] through the SHELXLE GUI (Göttingen, Germany) [29]. Due to the limited resolution and structural complexity of the model, soft restraints on bond lengths and angles of the host and guest molecules were applied using the GRADE webserver (Globar Phasing Ltd., Cambridge, UK) [42]. A GRADE dictionary for SHELXL contains target values and standard deviations for 1,2-distances (DFIX) and 1,3-distances (DANG), as well as restraints for planar groups (FLAT). Following a CSD search on structures containing SA-SA H-bonded dimer, SA molecules were restrained to be planar. Anisotropic displacement parameters were refined using the new rigid bond restraint (RIGU) implemented in the SHELXL program [43]; SIMU restraints were also applied where necessary. ISOR restrains were used on a handful of atoms to cure ill-defined ADPs. All $\mathrm{H}$-atoms of $\alpha-\mathrm{CD}$ and SA were placed geometrically and allowed to ride on the parent atoms. $\mathrm{H}$-atoms belonging to ordered water molecules were clearly visible in difference Fourier maps and their positions were refined subject to distance restraints. H-atoms belonging to disordered water molecules were not placed during refinement but were taken into account for the calculation of $F_{000}$ and derived properties. $U_{\text {iso }}(\mathrm{H})$ values were assigned in the range 1.2-1.5 times $U_{\text {eq }}$ of the parent atom. Details on the treatment of disorder are given in the discussion below and crystallographic 
details can be found in Table S1. CCDC 1437063-1437070 contain the supplementary crystallographic data for this paper. The data can be obtained free of charge from The Cambridge Crystallographic Data Center via www.ccdc.cam.ac.uk/getstructures.

\subsection{Disorder Modeling}

The structural refinement of $\alpha$-CD.SA was particularly challenging due to the heavy disorder of the guest and solvent molecules as well as one of the CD molecules. The disordered $\alpha-C D C$ molecule was modelled using a free variable which for the $90 \mathrm{~K}$ structure refines to a final value of $0.668(5)$ for the major component. The effect of disorder is to elongate the vase-like cluster (Figure 2). At $90 \mathrm{~K}$, a careful inspection of the difference Fourier maps permits the refinement of 33 positions of water molecules, which are distributed heterogeneously over the asymmetric unit and of which only two are fully occupied. For these two water molecules, $\mathrm{H}$-atoms could be located in difference Fourier maps.

The channel-type structure of $\alpha$-CD.SA favors the guest to be in the trans, planar conformation. The three-fold rotation axis, going through the center of the host, is incompatible with the guest molecular symmetry and imposes the presence of disorder, which was modelled as exemplified by $\alpha$-CD molecules B (Figure 7) as follows: electron density maps inside the $\alpha$-CD cavity show tetrahedral features, incompatible with a single SA molecule. The difference electron density peak $\mathrm{Q}(1)$, sitting on the 3-fold rotation axis, was assigned to the carbon atom $\mathrm{C}(1)$ of the carboxylic group. The coordinates of $\mathrm{C}(1)$ were constrained to make model building more straightforward. By using stereochemical restrains generated with the GRADE webserver [42] all other Q peaks were correctly assigned, i.e., $\mathrm{Q}(4)$ to $\mathrm{O}(1)$ and $\mathrm{O}(2), \mathrm{Q} 3$ to $\mathrm{C}(2), \mathrm{Q}(2)$ to $\mathrm{C}(4)$ (Figure 7). Finally, all atomic coordinates were freely refined. Two partially occupied SA molecules fit inside the disordered electron density; the negative PART instruction was used in the program SHELXL to exclude the generation of special position constraints and dissociate bonding to symmetry-generated atoms within the same PART group.

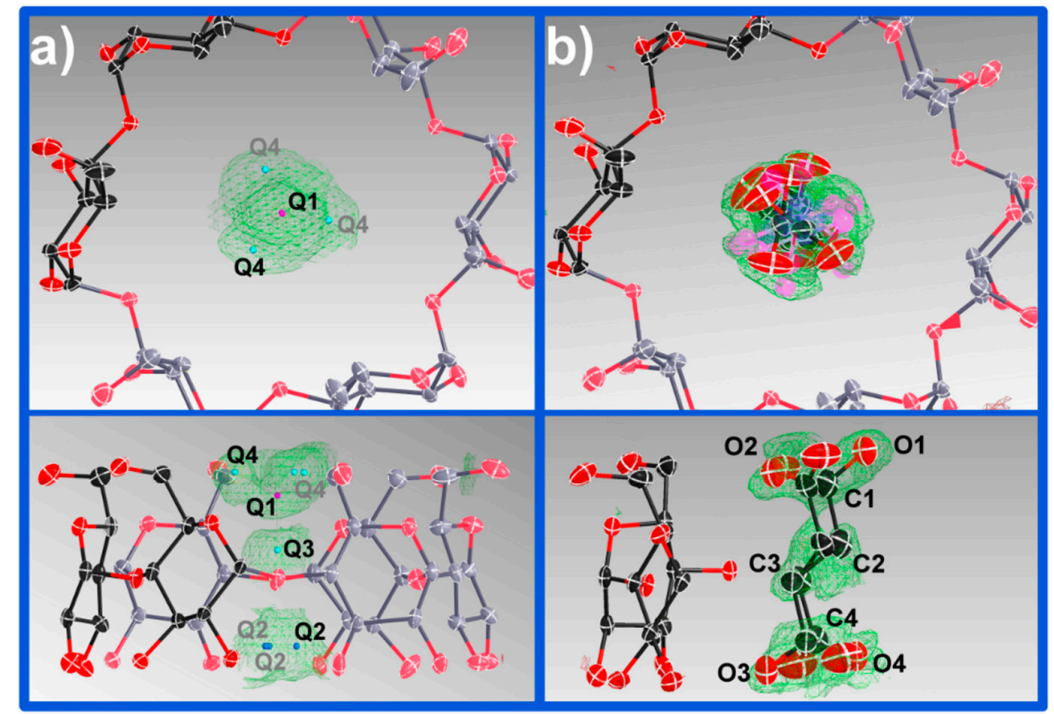

Figure 7. $F_{\mathrm{obs}}-F_{\text {calc }}$ maps in green showing the electron density before (a) and after (b) modeling SA inside $\alpha$-CD B molecule. The peaks Q1 and $3 \times(\mathrm{Q} 4)$ are forming a tetrahedron. H-atoms and the rest of the structure have been omitted for clarity. Displacement ellipsoids are drawn at the $50 \%$ probability level.

After several trials, the best refinement strategy consisted of gradually modeling the electron density of the host molecules and the reliably localized solvent peaks, followed by the guests, and finally the rest of the disordered solvent molecules. In order to avoid over-parametrization , 
site-occupancy factors of the disordered water molecules were fixed to the initially refined values. Those of the guest molecules were constrained to $1 / 6$ each.

\section{Conclusions}

The crystal structure of a 1:1 $\alpha$-CD.SA pseudododecahydrate inclusion complex has been elucidated using single-crystal X-ray diffraction. The complex crystallizes in a high-symmetry space group, previously unreported for $\alpha-\mathrm{CD}$ inclusion complexes. The structure exhibits a honeycomb arrangement of channels, in which the guest molecules are heavily disordered. The building block of the channels has been described as a vase-like cluster formed by a trimer of $\alpha$-CD molecules.

Structural disorder has been analyzed by means of a multi-temperature $\mathrm{X}$-ray diffraction experiment. Careful analysis of difference Fourier maps proved that the nature of disorder is, at least partially, dynamic. In the low-temperature regime disorder is attributed to thermal vibrations; above $210 \mathrm{~K}$ vibrations of water molecules accentuate the disorder and lead eventually to the decomposition of the crystal at ambient temperature.

Supplementary Materials: The following are available online at www.mdpi.com/2073-4352/6/1/2, Table S1: Crystallographic data of the multi-temperature structures of $\alpha$-CD.SA inclusion complex, Figure S1: Evolution of the normalized distances between dimers in $\alpha$-CD.SA. Distances were calculated based on the O(4) glycosidic planes using MERCURY, Figure S2: The solvent accessible void volume calculated using MERCURY (grid spacing $=0.2 \AA$; probe radius $=1.2 \AA$ ), Figure S3: $U_{11}, U_{22}$ and $U_{33}$ elements of the ADP tensor for selected atoms plotted as a function of temperature. The value of the ADPs were taken from the result file of the refinement of SHELX (res file). $\mathrm{O}(1)$ to $\mathrm{O}(6)$ are water molecules located in the interstices. The numbering suffix refers to the residue number in the structure.

Acknowledgments: The authors gratefully acknowledge funding from the German Science Foundation (DFG, Emmy Noether Grant to FPAF, FA 946/1-1) and technical support from Ulf Kahmann (Göttingen). The authors also wish to thank Werner F. Kuhs (Göttingen) for critical reading of the manuscript. The authors would like to thank anonymous Reviewers for helpful comments.

Author Contributions: Experimental investigations, data interpretation and manuscript writing were performed by Sofiane Saouane with the support of Francesca P. A. Fabbiani.

Conflicts of Interest: The authors declare no conflict of interest.

\section{References}

1. Irie, T.; Uekama, K. Pharmaceutical Applications of Cyclodextrins. III. Toxicological Issues and Safety Evaluation. J. Pharm. Sci. 1997, 86, 147-162. [CrossRef] [PubMed]

2. Uekama, K.; Hirayama, F.; Irie, T. Cyclodextrin Drug Carrier Systems. Chem. Rev. 1998, 98, $2045-2076$. [CrossRef] [PubMed]

3. Loftsson, T.; Brewster, M.E. Pharmaceutical Applications of Cyclodextrins. 1. Drug Solubilization and Stabilization. J. Pharm. Sci. 1996, 85, 1017-1025. [CrossRef] [PubMed]

4. Roux, M.; Perly, B.; Djedaïni-Pilard, F. Self-Assemblies of Amphiphilic Cyclodextrins. Eur. Biophys. J. 2007, 36, 861-867. [CrossRef] [PubMed]

5. Saenger, W. Cyclodextrin Inclusion Compounds in Research and Industry. Angew. Chemie Int. Ed. Engl. 1980, 19, 344-362. [CrossRef]

6. Saenger, W.; Jacob, J.; Gessler, K.; Steiner, T.; Hoffmann, D.; Sanbe, H.; Koizumi, K.; Smith, S.M.; Takaha, T. Structures of the Common Cyclodextrins and Their Larger Analogues-Beyond the Doughnut. Chem. Rev. 1998, 98, 1787-1802. [CrossRef] [PubMed]

7. Saenger, W.; Steiner, T. Cyclodextrin Inclusion Complexes: Host-Guest Interactions and Hydrogen-Bonding Networks. Acta Crystallogr. Sect. A 1998, 54, 798-805. [CrossRef]

8. Harata, K. Structural Aspects of Stereodifferentiation in the Solid State. Chem. Rev. 1998, 98, $1803-1827$. [CrossRef] [PubMed]

9. Granero-García, R.; Lahoz, F.J.; Paulmann, C.; Saouane, S.; Fabbiani, F.P.A. A Novel Hydrate of A-Cyclodextrin crystallized under High-Pressure Conditions. CrystEngComm 2012, 14, 8664-8670. [CrossRef] 
10. Allen, F.H. The Cambridge Structural Database: A Quarter of a Million Crystal Structures and Rising. Acta Crystallogr. Sect. B Struct. Sci. 2002, 58, 380-388. [CrossRef]

11. Zeikus, J.G.; Jain, M.K.; Elankovan, P. Biotechnology of Succinic Acid Production and Markets for Derived Industrial Products. Appl. Microbiol. Biotechnol. 1999, 51, 545-552. [CrossRef]

12. Lisnyak, Y.V.; Martynov, A.V.; Baumer, V.N.; Shishkin, O.V.; Gubskaya, A.V. Crystal and Molecular Structure of $\beta$-Cyclodextrin Inclusion Complex with Succinic Acid. J. Incl. Phenom. Macrocycl. Chem. 2007, 58, 367-375. [CrossRef]

13. Del Valle, E.M.M. Cyclodextrins and Their Uses: A Review. Process Biochem. 2003, 39, 1033-1046. [CrossRef]

14. Harata, K.; Uekama, K.; Otagiri, M.; Hirayama, F. Crystal Structures Pentahydrate of a-Cyclodextrin-3-Lodopropionic Acid (1:1) Complex and Hexakis(2, 3, 6-Tri-O-Methyl)-a-CyclodextrinLodoacetic Acid (1:1) Complex Monohydrate. Jpn. Chem. Soc. J. 1983, 2, 173-180.

15. Tokuoka, R.; Abe, M.; Matsumoto, K.; Shirakawa, K.; Fujiwara, T.; Tomita, K. Structure of the $\alpha$-Cyclodextrin $(\alpha-C D)$ Inclusion Complex with the Potassium Salt of $\gamma$-Aminobutyric Acid (GABA). Acta Crystallogr. Sect. B Struct. Crystallogr. Cryst. Chem. 1981, 37, 445-447. [CrossRef]

16. Sicard-Roselli, C.; Perly, B.; le Bas, G. The Respective Benefits of X-ray Crystallography and NMR for the Structural Determination of the Inclusion Complex Between Butyl-Isothiocyanate and Alpha-Cyclodextrin. J. Incl. Phenom. Macrocycl. Chem. 2001, 39, 333-337. [CrossRef]

17. Ding, J.; Steiner, T.; Saenger, W. Structure of the $\gamma$-cyclodextrin-1-propanol- $17 \mathrm{H}_{2} \mathrm{O}$ Inclusion Complex. Acta Crystallogr. Sect. B Struct. Sci. 1991, 47, 731-738. [CrossRef]

18. Kamitori, S.; Hirotsu, K.; Higuchi, T. Crystal and Molecular Structures of Double Macrocyclic Inclusion Complexes Composed of Cyclodextrins, Crown Ethers, and Cations. J. Am. Chem. Soc. 1987, 109, 2409-2414. [CrossRef]

19. Steiner, T.; Saenger, W. Channel-Type Crystal Packing in the Very Rare Space Group P4212 with $Z^{\prime}=3 / 4$ : Crystal Structure of the Complex $\gamma$-Cyclodextrin-Methanol-n-Hydrate. Acta Crystallogr. Sect. B Struct. Sci. 1998, 54, 450-455. [CrossRef]

20. Chatziefthimiou, S.; Yannakopoulou, K.; Mavridis, I.M. $\beta$-Cyclodextrin trimers enclosing an unusual organization of the guest: The inclusion complex of $\beta$-cyclodextrin/4-pyridinealdazine. CrystEngComm 2007, 9, 976-979. [CrossRef]

21. Cheng, X.; Lu, Z.; Li, Y.; Wang, Q.; Lu, C.; Meng, Q. An interesting molecular-assembly of $\beta$-cyclodextrin pipelines with embedded hydrophilic nickel maleonitriledithiolate. Dalton Trans. 2011, 40, 11788-11794. [CrossRef] [PubMed]

22. Caira, M.; Dodds, D.; Nassimbenni, L.R. Diverse modes of guest inclusion in a $\beta$-cyclodextrin: X-ray structural and thermal characterization of a 4:3 $\beta$-cyclodextrin-cyclizine complex. Supramol. Chem. 2001, 13, 61-70. [CrossRef]

23. Liu, Y.; Zhong, R.Q.; Zhang, H.Y.; Song, H.B. A unique tetramer of 4:5 $\beta$-cyclodextrin-ferrocene in the solid state. Chem. Commun. 2005, 17, 2211-2213.

24. Harada, A.; Li, J.; Kamachi, M.; Kitagawa, Y.; Katsube, Y. Structures of Polyrotaxane Models. Carbonhydr. Res. 1998, 305, 127-129. [CrossRef]

25. Caira, M.; Bourne, S.; Mzondo, B. Cyclodextrin inclusion complexes of the antioxidant $\alpha$-lipoic acid. Acta Crystallogr. Sect. A Found. Cryst. 2014, 70, C992. [CrossRef]

26. Saenger, W. Nature and Size of Included Guest Molecule Determines Architecture of Crystalline Cyclodextrin Host Matrix. Isr. J. Chem. 1985, 25, 43-50. [CrossRef]

27. Chacko, K.K.; Saenger, W. Topography of Cyclodextrin Inclusion Complexes. 15. Crystal and Molecular Structure of the Cyclohexaamylose-7.57 Water Complex, Form III. Four- and Six-Membered Circular Hydrogen Bonds. J. Am. Chem. Soc. 1981, 103, 1708-1715. [CrossRef]

28. Gruene, T.; Hahn, H.W.; Luebben, A.V.; Meilleur, F.; Sheldrick, G.M. Refinement of Macromolecular Structures against Neutron Data with SHELXL2013. J. Appl. Crystallogr. 2014, 47, 462-466. [CrossRef] [PubMed]

29. Hübschle, C.B.; Sheldrick, G.M.; Dittrich, B. ShelXle: A Qt Graphical User Interface for SHELXL. J. Appl. Crystallogr. 2011, 44, 1281-1284. [CrossRef] [PubMed]

30. Macrae, C.F.; Bruno, I.J.; Chisholm, J.A.; Edgington, P.R.; McCabe, P.; Pidcock, E.; Rodriguez-Monge, L.; Taylor, R.; van de Streek, J.; Wood, P.A. Mercury CSD 2.0-New Features for the Visualization and Investigation of Crystal Structures. J. Appl. Crystallogr. 2008, 41, 466-470. [CrossRef] 
31. Cruz-Cabeza, A.J.; Day, G.M. Structure prediction, disorder and dynamics in a DMSO solvate of carbamazepine. Phys. Chem. Chem. Phys. 2011, 13, 12808-12816. [CrossRef] [PubMed]

32. Nyman, J.; Day, G.M. Static and lattice vibrational energy differences between polymorphs. CrystEngComm 2015, 17, 5154-5165. [CrossRef]

33. Price, S.L. Predicting crystal structures of organic compounds. Chem. Soc. Rev. 2014, 43, $2098-2111$. [CrossRef] [PubMed]

34. Neumann, M.A.; van de Streek, J.; Fabbiani, F.P.A.; Hidber, P.; Grassmann, O. Combined Crystal Structure Prediction And High-Pressure Crystallization in Rational Pharmaceutical Polymorph Screening. Nat. Commun. 2015, 6, 7793. [CrossRef] [PubMed]

35. Merrill, L.; Bassett, W.A. Miniature Diamond Anvil Pressure Cell for Single Crystal X-ray Diffraction Studies. Rev. Sci. Instrum. 1974, 45, 290-294. [CrossRef]

36. Piermarini, G.J.; Block, S.; Barnett, J.D.; Forman, R.A. Calibration of the Pressure Dependence of the R1 Ruby Fluorescence Line to 195 Kbar. J. Appl. Phys. 1975, 46, 2774-2780. [CrossRef]

37. Kottke, T.; Stalke, D. Crystal Handling at Low Temperatures. J. Appl. Crystallogr. 1993, 26, 615-619. [CrossRef]

38. Bruker-AXS Inc. SAINT Version 8.34A; Bruker-AXS Inc: Madison, WI, USA, 2013.

39. Sheldrick, G.M. SADABS Version 2012/1, 1; Bruker-AXS Inc: Madison, WI, USA, 2012.

40. Sheldrick, G.M. A Short History of SHELX. Acta Crystallogr. A 2008, 64, 112-122. [CrossRef] [PubMed]

41. Sheldrick, G.M. Crystal Structure Refinement with SHELXL. Acta Crystallogr. Sect. C Struct. Chem. 2015, 71, 3-8. [CrossRef] [PubMed]

42. Grade Web Server, Global Phasing Ltd. Grade Web Server. Available online: http://grade.globalphasing.org/ (accessed on 15 January 2015).

43. Thorn, A.; Dittrich, B.; Sheldrick, G.M. Enhanced Rigid-Bond Restraints. Acta Crystallogr. Sect. A Found. Crystallogr. 2012, 68, 448-451. [CrossRef]

(C) 2015 by the authors; licensee MDPI, Basel, Switzerland. This article is an open access article distributed under the terms and conditions of the Creative Commons by Attribution (CC-BY) license (http://creativecommons.org/licenses/by/4.0/). 